\title{
IRF-5 Expression in Myeloid Cells Is Required for Splenomegaly in $L$. donovani Infected Mice
}

\author{
Linh Thuy Mai, Mélina Smans, Sasha Silva-Barrios, Aymeric Fabié and Simona Stäger* \\ Centre Armand-Frappier Santé Biotechnologie, Institut National de la Recherche Scientifique (INRS), Laval, QC, Canada
}

Persistent Leishmania donovani infection is characterized by chronic inflammation, immune suppression, and splenomegaly. We have previously reported that the transcription factor interferon regulatory factor 5 (IRF-5) is largely responsible for inducing the inflammatory response and maintaining protective Th1 cells following $L$. donovani inoculation in mice. However, the cellular source responsible for these effects is yet unknown. In this study, we investigated the role of IRF-5 in myeloid cells during experimental visceral leishmaniasis (VL). First, we show that the LysM-Cre mouse model is not suited for investigating gene expression in splenic myeloid cells during experimental VL. Using the Cd11c-Cre mouse model, we demonstrate that IIf5 expression in CD11 $\mathrm{c}^{+}$ cells (monocytes, dendritic cells, activated macrophages) is essential for inducing splenomegaly and for recruiting myeloid cells to the spleen, but it is not required for the development or maintenance of parasite-specific IFN $\gamma$-producing CD4 T cells. CD11C-specific Irf5 ${ }^{-1-}$ mice are more resistant to $L$. donovani infection, suggesting that the induction of splenomegaly is detrimental to the host.

Keywords: Leishmania, IRF5, myeloid cells, splenomegaly, Th1, LysM-cre

\section{INTRODUCTION}

The Leishmaniasis are a set of vector-borne parasitic diseases that affect an estimated 12 million people worldwide. Disease clinical manifestations range from self-healing cutaneous lesions to life-threatening visceral infection. The visceral form of the disease has a fatality rate of $75-95 \%$ if left untreated (1) and is characterized by weight loss, irregular bouts of fever, anemia, hepatosplenomegaly, hypergamaglobulinemia, and immunosuppression. Experimental infection of mice with Leishmania donovani, a causative agent of visceral leishmaniasis (VL), also results in hepatosplenomegaly, but it is not fatal (2). In mice, parasite growth in the liver is controlled by protective CD4 and CD8 T cell responses producing IFN $\gamma$ (3). In contrast, infection persists in the spleen and the bone marrow.

The chronic stage of experimental VL in the spleen is characterized by a chronic inflammatory environment, with all the consequences that this entails: tissue disruption, hypoxia, and immunoregulatory responses $(2,4,5)$. Leishmania parasites survive very well in this environment, whereas protective $\mathrm{T}$ cell responses are inhibited in various ways. For instance, at $\mathrm{d} 28 \mathrm{p}$.i., CD8 $\mathrm{T}$ cells are dysfunctional and exhausted $(6,7)$ and CD4 T cells fail to expand (8), die by TRAIL-mediated apoptosis (9), and are suppressed by IL-10 (10-13) and by myeloid-derived suppressor cells (14). 
Splenomegaly and chronic inflammation are associated with parasite persistence during chronic VL. Several cell populations contribute to splenomegaly, but myeloid cells, in particular, are progressively recruited to the spleen over the course of infection (14). Indeed, $L$. donovani induces the heightened release from the bone marrow of inflammatory monocytes (15). These cells display a regulatory phenotype and are more permissive to infection, favoring parasite growth and persistence (14-16). The inflammatory response during VL appears to require the activation of the transcription factor Interferon Regulatory Factor 5 (IRF-5). IRF-5 function has been mainly described in antigen-presenting cells, where it promotes the transcriptional activation of genes encoding for IFN-I and proinflammatory cytokines, such as TNF, IL-12, and IL-6 $(17,18)$. In human, IRF-5 polymorphisms are associated with various autoimmune inflammatory disorders (19-22). In mice infected with L. donovani, IRF-5 governs the inflammatory response (23), but it is also responsible for CD4 T cell death during chronic infection (9). Indeed, Irf5 $5^{-/-}$mice fail to develop splenomegaly and to develop protective Th1 responses following L. donovani infection (23). Nevertheless, the cellular source required for promoting IRF-5-dependent inflammation and sustaining Th1 responses during experimental VL is yet unknown.

In this study, we investigated the role of IRF- 5 in myeloid cells following $L$. donovani infection in mice. We show that LysMCre mice are not a good model for investigating gene expression in splenic myeloid cells during experimental VL. We also demonstrate that Irf5 expression in $\mathrm{CD}_{11} \mathrm{c}^{+}$cells is essential for inducing splenomegaly, but it is not required for the development or maintenance of parasite-specific IFN $\gamma$-producing CD4 T cells.

\section{MATERIALS AND METHODS}

\section{Mice and Parasites}

B6.129S7-Rag1 ${ }^{\text {tm } 1 M o m}$ and cre recombinase-expressing mice were purchased from The Jackson Laboratory. Mice with a targeted Irf5 mutation in myeloid and in $\mathrm{CD} 11 \mathrm{c}^{+}$cells were generated by crossing Irff $5^{\text {flox/flox }}$ mice with mice expressing the cre-recombinase under the LysM and the CD11c promoter, respectively. All mice were housed at the INRS animal facility under specific pathogen-free conditions and used at 6-10 weeks of age. Leishmania donovani (strain LV9) were maintained by serial passage in B6.129S7-Rag1 $1^{\text {tm1Mom }}$ mice; amastigotes were isolated from the spleen of infected animals (24). Mice were infected by injecting $2 \times 10^{7}$ amastigotes intravenously via the lateral tail vein. Splenic parasite burden were determined by examining methanol-fixed, Giemsa stained tissue impression smears. Data are presented as Leishmania Donovani Units (LDU) (25).

\section{Ethic Statement}

Experiments involving mice were carried out under protocols approved by the Comité Institutionnel de Protection des Animaux of the INRS-Institut Armand Frappier (1510-02, 160202). These protocols respect procedure on good animal practice provided by the Canadian Council on animal care.

\section{Flow Cytometry}

Mice were euthanized at indicated time points. Mononuclear cells were purified from the liver and CD4 T cell responses were analyzed as previously described (14). Briefly, hepatic mononuclear cells were restimulated with bone marrow-derived dendritic cells, pulsed with fixed parasites, and directly incubated at $37^{\circ} \mathrm{C}$ in the presence of $1 / 1000$ Brefeldin A (GolgiPlug ${ }^{\mathrm{TM}}$, $\mathrm{BD}$ Biosciences). Cells were then stained with anti-CD4FITC (BD Pharmingen ${ }^{\mathrm{TM}}$, clone GK15), anti-CD3-BV421 (BD Biosciences, clone 14S-2C11), followed by anti-IFN- $\gamma$-APC (BD Pharmingen ${ }^{\mathrm{TM}}$, clone XMG1.2) after permeabilization with $0.1 \%$ saponin. Myeloid cells were stained with antiCD11b-Pacific Blue (BD Horizon ${ }^{\mathrm{TM}}$, clone MI/70), anti-MHCII-FITC (BD PharmingenTM, clone 2G9), anti-Ly6C-PerCP (Biolegend, clone HK1.4), anti-Ly6G-PE (Biolegend, clone 1A8), anti-F4/80-PECy7 (Biolegend, clone BM8), and anti-CD11cAPC (eBioscience, clone N418). Flow cytometric analysis was performed with a BD LSRFortessa cell analyzer (Becton Dickinson). Samples were analyzed with Flowjo software.

\section{Enrichment of Splenic Myeloid Cells}

$\mathrm{CD}_{11} \mathrm{~b}^{+}$cells were purified using magnetic cell sorting (MACS) from spleens of infected and naïve mice previously digested with collagenase D, following manufacturer's instructions (Miltenyi Biotec). The purity of the samples comprise between 90 and $93 \%$.

\section{Real-Time PCR Analysis}

Real-time PCR (Stratagene mx3005p Real time PCR System) was used to analyze transcripts levels of HPRT, HIF- $1 \alpha$, and IRF5. Total RNA was insolated using RNeasy (Qiagen) to perform real-time RT-PCR. cDNA was prepared using $500 \mathrm{ng}$ of total RNA using High Capacity cDNA Reverse Transcription Kit (Bio Rad). Real time PCR was performed using standard cycle of amplification. All PCRs were carried out with the Stratagene mx3000p real-time PCR system. Irf5, Hprt, and Hif1a were amplified using primers as previously described $(9,14)$. Data were normalized to HPRT and expressed as fold increase to naive controls.

\section{Statistical Analysis}

Data were analyzed using Graphpad Prism (GraphPad Software). Statistical significance was assessed using two-way ANOVA. Differences were considered to be statistically significant when $p<0.05$. All experiments were conducted independently at least three times.

\section{RESULTS}

\section{Irf5-Deletion in Myeloid Cells Does Not Affect the Hepatic and Splenic Parasite Burden}

We have previously reported that the transcription factor IRF5 majorly contributes to the inflammatory response following Leishmania donovani infection in mice (23). Indeed, L. donovaniinfected Irf5 ${ }^{-/}$mice not only failed to develop splenomegaly, but also displayed severely impaired parasite-specific Th1 responses. This resulted in higher hepatic parasite burden, but 
no differences were observed in the spleen (23). In the present study, we sought to identify the contribution of myeloid cellderived IRF-5 expression to the immune response against $L$. donovani. To this end, we generated myeloid cell-specific Irf5 ${ }^{-/-}$ mice by crossing Irf5 flox/flox with LysM-Cre mice and infected $\mathrm{Cre}^{+}$(myeloid cells are IRF-5 deficient) and $\mathrm{Cre}^{-}$(myeloid cells are IRF-5 sufficient) littermates with $L$. donovani. Because $\operatorname{Irf5} 5^{-/-}$were shown to have a spontaneous mutation in Dock2 that affected pDC and B cell development (26), we generated new Irf5 $5^{-/-}$mice by crossing Irf $5^{\text {flox/flox }}$ with $C M V$-Cre mice. These mice were used as total Irf5 $5^{-/-}$controls in all experiments. First, we assessed the hepatic and splenic parasite burden. As expected, $L$. donovani infection in the liver was significantly exacerbated in Irff flox/flox $-\mathrm{CMV}$-Cre ${ }^{+}$mice compared to infected Irff flox/flox - LysM-Cre ${ }^{-}$control mice (Figure 1A). In contrast, no differences were observed between the Irf5 flox/flox - LysM$\mathrm{Cre}^{+}$and $\mathrm{Cre}^{-}$groups. As previously reported (23), the absence of IRF-5 expression in all cells did not affect the splenic parasite load (Irf5 flox/flox $\left.-\mathrm{CMV}-\mathrm{Cre}{ }^{+}\right)$; moreover, $\mathrm{Cre}{ }^{+}$mice had similar parasite numbers in the spleen than their $\mathrm{Cre}^{-}$ counterpart (Figure 1B).

\section{Myeloid-Specific Irf5-/- Mice Develop Similar Th1 Responses Than IRF-5-Sufficient Mice}

A hallmark of $\operatorname{Irf5} 5^{-/}$mice is their incapacity to generate strong Th1 responses following L. donovani infection (23). Krausgruber et al. have also reported that IRF-5 is required to promote inflammatory macrophages and induce Th1 responses (27). Hence, we evaluated parasite-specific IFN $\gamma^{+} \mathrm{CD} 4$ cell responses at various time points of $L$. donovani infection in total Irf5 $5^{-/-}$ $\left(\mathrm{CMV}-\mathrm{Cr} \mathrm{e}^{+}\right)$, in myeloid cell-specific $\operatorname{Irf5} 5^{-/-}\left(\mathrm{Cre}^{+}\right)$, and in IRF-5 sufficient $\left(\mathrm{Cr}^{-}\right)$mice. As shown in Figure $\mathrm{IC}$ and in agreement with the literature (23), in $\mathrm{Cr}^{-}$control mice, hepatic Th1 responses developed quite slowly from d14 p.i. on following

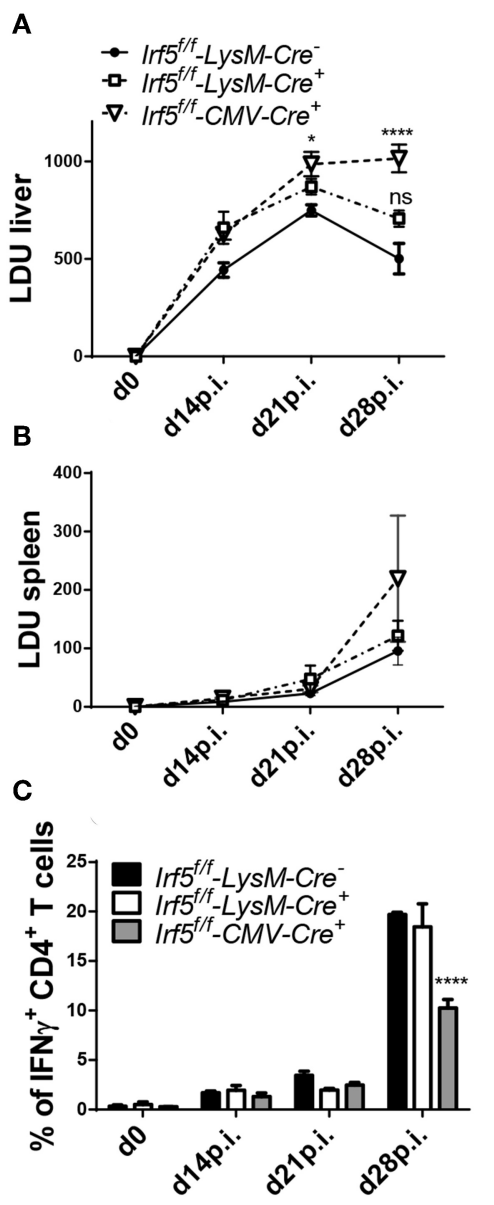

D

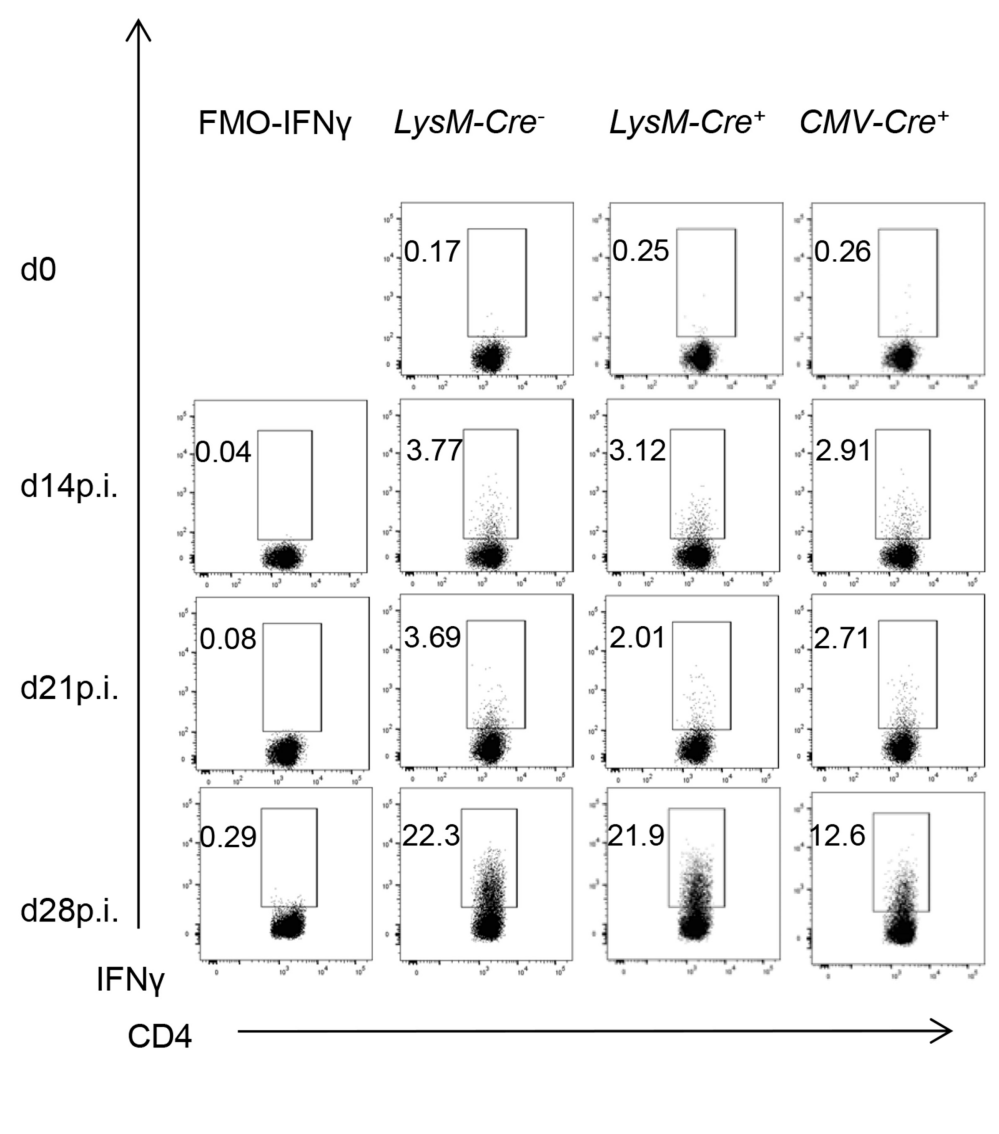

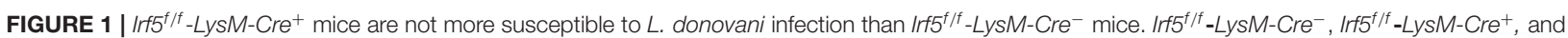
Irff $/ / f-C M V-C r e+$ mice were infected with $L$. donovani and euthanized at d14, 21, and 28 p.i. Leishmania Donovan Units (LDU) were calculated from hepatic (A) and splenic (B) impression smears for the three group of mice. Graphs show representative scatter plots (C) and percentages (D) of CD4 T cells producing IFN- $\gamma$ following $L$. donovani infection in the liver from three groups of mice. Error bars indicate mean $\pm \mathrm{SEM}, N=4$, one of 5 independent experiments is shown; ns, not significant, ${ }^{*} p<0.05,{ }^{* \star * *} p<0.0001$. 

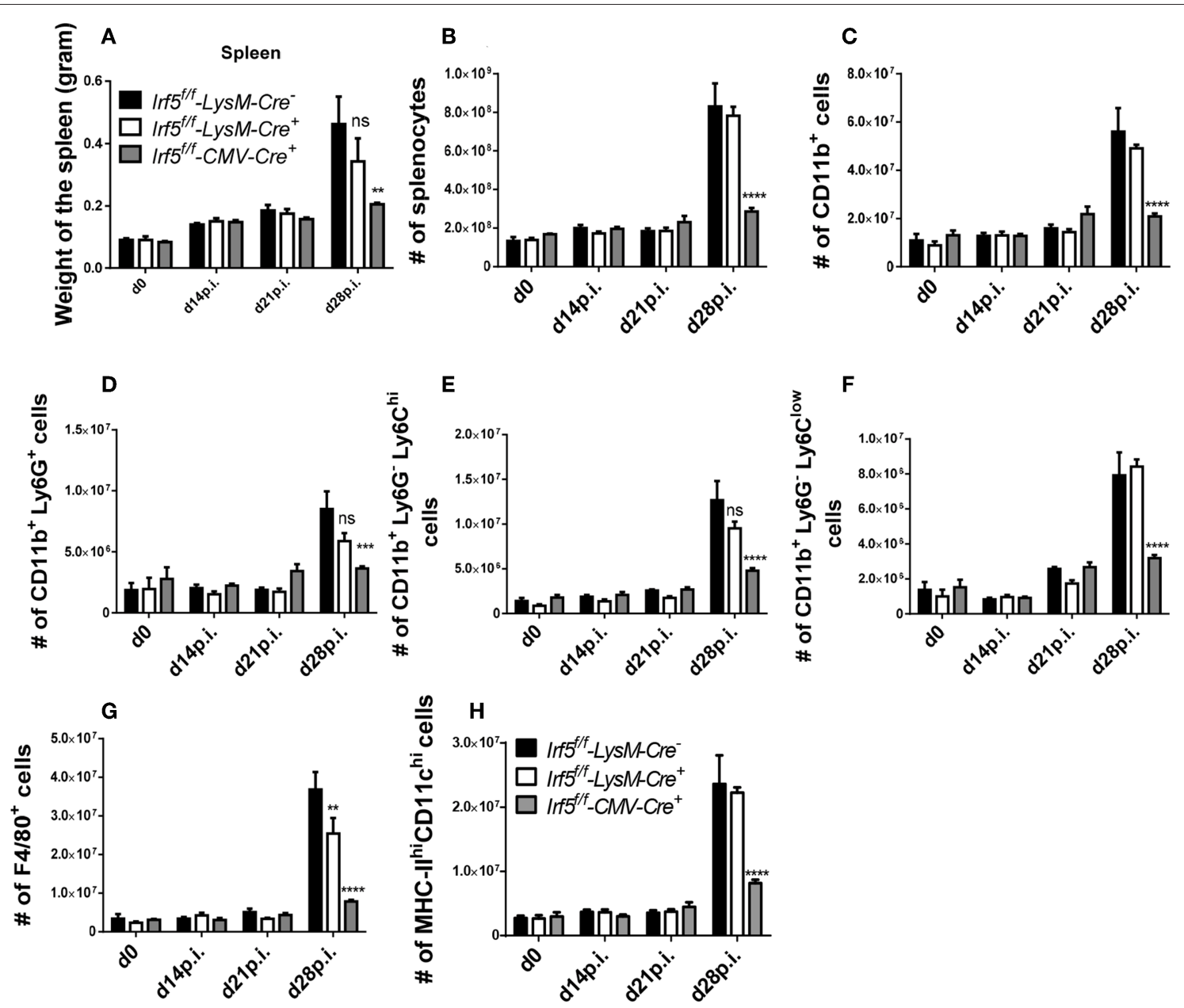

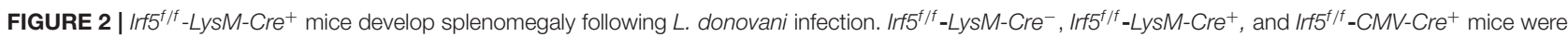
infected with L. donovani and sacrificed at d14, d21, and d28 p.i. Graphs show spleen weights (A), number of splenocytes (B) and absolute number of splenic $\mathrm{CD}_{11 \mathrm{~b}}{ }^{+}$cells $(\mathbf{C})$ in naïve and infected mice over the course of infection. Splenocytes were stained with different surface markers; neutrophils were excluded before analyzing monocytes and monocyte-derived cells. Graphs show absolute number of Ly6G+ neutrophils (D), Ly6Chigh monocytes (E), Ly6Clow monocytes (F), F4/80+ cells $(\mathbf{G})$, and MHC-II high $C D 11 c^{\text {high }}$ cells $\mathbf{( H )}$. Data is shown as the mean $\pm \mathrm{SEM}, N=3-4$, one of 5 independent experiments is shown, ns, not significant, ${ }^{\star *} p<$ $0.01,{ }^{\star \star *} p<0.001,{ }^{\star * \star *} p<0.0001$.

L. donovani inoculation and peaked between $\mathrm{d} 21$ and 28 p.i., when about $20 \%$ of the CD $4 \mathrm{~T}$ cells expressed IFN $\gamma$. Similarly to Irf5 ${ }^{-/-}$mice (23), infected CMV-Cre ${ }^{+}$mice generated defective Th1 responses and only about $10 \%$ of the CD4 T cells in the liver were $\mathrm{IFN} \gamma^{+}$at d 28 p.i. (Figures 1C,D; gating strategy is shown in Supplemental Figure 1A). Interestingly, myeloidspecific Irf5 $5^{-/}$mice had similar frequencies of IFN $\gamma^{+}$CD4 T cells to the $\mathrm{Cre}^{-}$controls, suggesting that IRF-5 expression in myeloid cells is not required for Th1 development in L. donovani infected mice.

\section{Irf5 flox/flox - LysM-Cre ${ }^{+}$Mice Develop Splenomegaly}

Next, we wanted to determine whether IRF-5 expression in myeloid cells was at all required to induce splenomegaly that is typically present following $L$. donovani infection.
As expected, the splenic weight (Figure 2A) and cellularity (Figure 2B) constantly increased over the course of L. donovani infection in $\mathrm{Cre}^{-}$controls. In contrast, spleen of infected $\mathrm{CMV}-\mathrm{Cre}^{+}$mice had a significantly lower weight (Figure 2A) and cell count (Figure 2B), in agreement with our previously published results using $\operatorname{Irf5} 5^{-/-}$(23). Because myeloid cells are increasingly recruited to the spleen during $L$. donovani infection $(14,15)$, we analyzed myeloid cell recruitment over the course of infection in the three groups of mice. As shown in Figure 2C, the number of $\mathrm{CD}_{11 b^{+}}$cells present in the spleen of $\mathrm{Cre}^{-}$mice progressively increased until d28 p.i.; comparable myeloid cell numbers were also observed in the spleen of cell-specific Irf5 $5^{-/}$mice (Figure 2C). In contrast, splenic myeloid cell numbers in total IRF-5-deficient mice (CMV-Cre) did not substantially vary over the course of disease and were significantly lower than in the other two groups 
(Figure 2C). We obtained similar results when we analyzed the various myeloid cell subpopulations. In naïve mice and in L. donovani-infected mice until d14p.i., splenic myeloid cells can be clearly subdivided into neutrophils $\left(\mathrm{CD} 11 \mathrm{~b}^{\text {hi }} \mathrm{Ly}_{6 \mathrm{G}}{ }^{+}\right.$), inflammatory monocytes (CD11b ${ }^{\text {hi }}$ Ly6G ${ }^{-}$Ly6C $^{\text {hi }}$ ), nonclassical monocytes (CD11b ${ }^{\text {hi }}$ Ly6G $\mathrm{G}^{-}$Ly6 $\mathrm{C}^{\text {lo/int }}$ ), macrophages $\left(\mathrm{CD} 11 \mathrm{~b}^{+} \mathrm{F} 4 / 80^{+} \mathrm{MHCII}^{\mathrm{lo}}\right)$, and conventional dendritic cells $\left(\mathrm{CD} 11 \mathrm{c}^{\mathrm{hi} M H C I I^{\text {int }} / \mathrm{hi}}\right.$ ) (gating strategies for all myeloid cell populations are in Supplemental Figure 1B). However, during chronic $L$. donovani infection these populations are much less clearly defined. With exception of neutrophils, which remain CD $11 b^{\text {hi }} \mathrm{Ly}_{6 \mathrm{G}}{ }^{+}$, at chronic stages of disease monocytes and monocytes-derived cells, such as macrophages and dendritic cells, acquire very similar markers, namely CD11b, MHCII, CD11c, and F4/80 $(14,15)$, which makes it very difficult to unmistakably differentiate dendritic cells and macrophages from monocytes and from each other. As observed for total myeloid cells, the number of neutrophils (Figure 2D), inflammatory monocytes (Figure 2E), non-classical monocytes (Figure 2F),

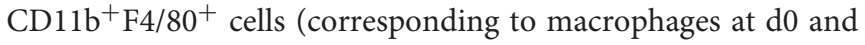
14p.i. and monocytes, macrophages and DCs at d21 and 28) (Figure 2G), and CD11 $\mathrm{c}^{\text {hi MHCII }}{ }^{\text {hi }}$ (DCs at d0 and $14 \mathrm{p} . \mathrm{i}$ and DC and inflammatory monocytes at $\mathrm{d} 21$ and 28p.i) (Figure 2H) dramatically increased during chronic stages of infection in IRF-5-sufficient control mice. As expected, the numbers of splenic neutrophils, monocytes, macrophages and DCs did not substantially change over the course of infection in $C M V$ $\mathrm{Cre}^{+}$mice and were significantly lower than those observed in $\mathrm{Cr}^{-}$mice (Figures 2D-H). Surprisingly, no significant differences were observed in the numbers of splenic myeloid cell populations, with exception of $\mathrm{F} 4 / 80^{+}$cells that were slightly fewer in cell-specific $\operatorname{Irf} 5^{-/-}$mice at d28p.i. when compared with IRF-5 sufficient control mice (Figures 2D-H). No differences were observed among the three groups of mice in the percentage of the various myeloid cell populations (data not shown), suggesting that the defect in recruitment observed in IRF-5-deficient mice at d 28 p.i. was not confined to myeloid cells.

Taken together, our results imply that IRF-5 expression in myeloid cells is not required for promoting inflammatory cell infiltration, Th1 responses, and splenomegaly during chronic experimental VL.

\section{LysM-Cre- Fails to Delete Irf5 in Splenic Myeloid Cells During Chronic VL}

Although LysM-Cre mice are the model of choice to delete specific genes in myeloid cells, we were puzzled by the fact that deletion of Irf5 in myeloid cells did not have any effect at all on the immune response to L. donovani or on the course of infection. Hence, we suspected that the cre recombinase had low deletion efficiency in splenic cells expressing lysozyme 2 in our particular infection model. Hence, we analyzed Irf5 expression by qPCR in purified splenic myeloid cells in L. donovani infected $\mathrm{Cre}^{+}$and $\mathrm{Cre}^{-}$mice. As a control, we also measured $\operatorname{Irf} 5$ mRNA expression levels in $\mathrm{CD} 1 \mathrm{~b}^{+}$cells from L. donovani infected Irf5-CMV-Cre ${ }^{+}$ mice, which, as expected, failed to upregulate Irf5 (Figure 3A).

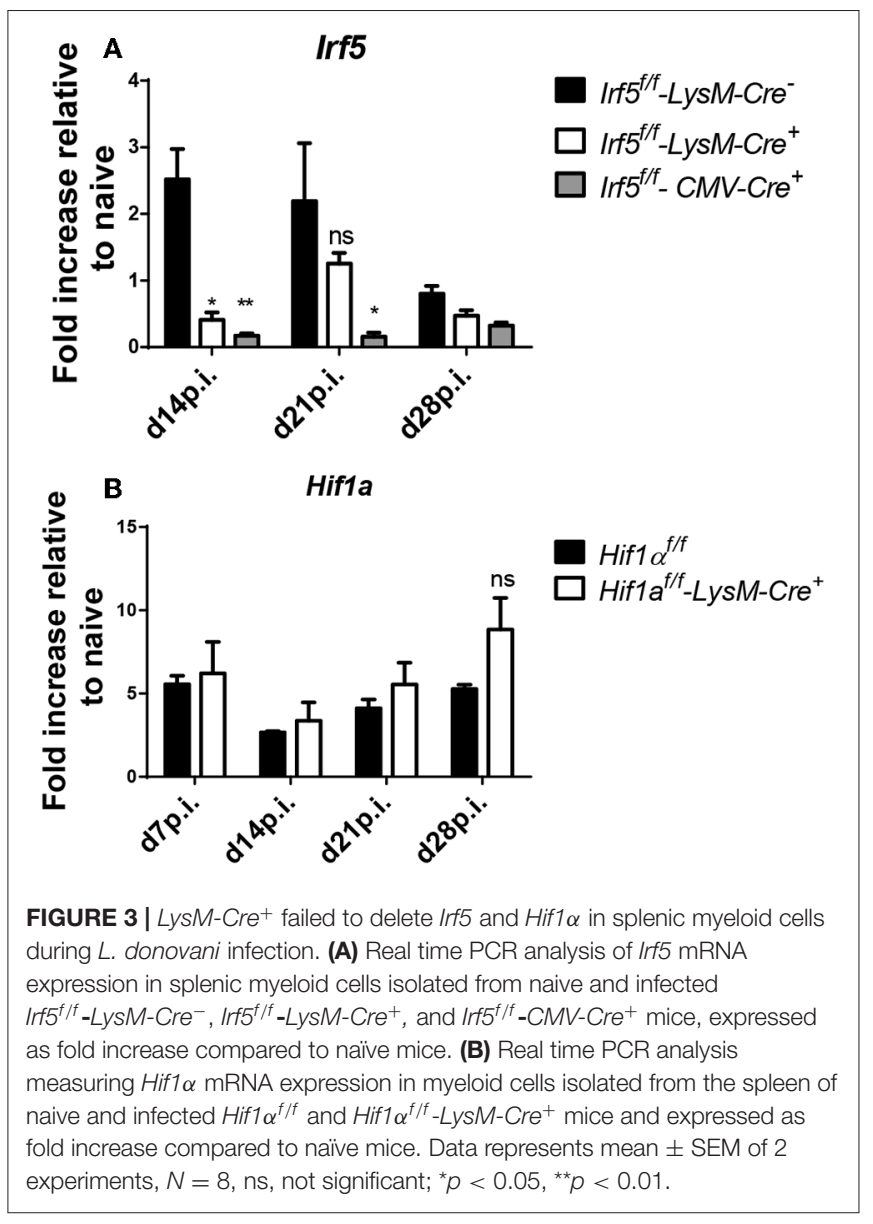

Significant deletion in $\mathrm{CD}_{11} \mathrm{~b}^{+}$cells was only achieved at $\mathrm{d} 14$ p.i. (Figure 3A); however, no difference in Irf5 mRNA expression was noted at $\mathrm{d} 21$ between $\mathrm{Cr}^{+}$and $\mathrm{Cr}^{-}$mice (Figure 3A). To exclude the possibility that this lack of deletion was associated with our particular target gene, Irf5, or our Irf5 flox/flox mice, we tested cre recombinase efficiency in splenic myeloid cells in a different mouse model, using a different target gene, namely Hif1a. Like for Irf5, we analyzed Hifla mRNA expression in purified splenic myeloid cells over the course of L. donovani infection in Hifla $a^{\text {flox/flox }}-\mathrm{LysM-Cre^{+ }}$ and $\mathrm{Cr}^{-}$mice. As shown in Figure 3B, no differences were observed in Hifla expression in splenic $\mathrm{CD}_{11 b^{+}}$cells from both groups of mice at any time point of infection, suggesting that the LysM-Cre model cannot be used to delete genes in splenic myeloid cells in the context of experimental VL.

\section{Myeloid Cell-Derived IRF-5 Promotes Splenomegaly but Is Not Required to Induce Th1 Responses During L. donovani Infection}

We next turned our attention to another model that we successfully used in the past to delete Hifla in splenic myeloid cells during L. donovani infection (14), namely knock-in mice 
expressing the cre recombinase under the CD11c promoter. This model can be used for experimental VL infection, since most splenic myeloid cells, excluding neutrophils, express CD11c during chronic infection and the deletion efficiency is much higher than that achieved with LysM-Cre mice (14). Thus, we generated $\mathrm{Irff} \mathrm{flox}^{\mathrm{fllox}}-\mathrm{Cd} 1 \mathrm{Cc}-\mathrm{Cre}^{+}$and $\mathrm{Cre}^{-}$mice and infected them with $L$. donovani. First we investigated the development of IFN $\gamma^{+} \mathrm{CD} 4 \mathrm{~T}$ cell responses in the liver, as those were mostly affected in total Irf5 $5^{-/}$mice following $L$. donovani infection. Surprisingly and in disagreement with the literature, we found that Irf5 deletion in $\mathrm{CD}_{11 c^{+}}$cells did not affect the Th1 immune response; indeed, comparable frequencies of IFN $\gamma^{+} \mathrm{CD} 4 \mathrm{~T}$ cells were observed at various time points after infection in the liver of both groups of mice (Figures 4A,B; gating strategy in Supplemental Figure 1A). Similarly, no differences were observed in the frequency of splenic IFN $\gamma^{+}$CD4 T cells between both groups of mice (Supplemental Figure 2).

However, Irf5 flox/flox $-C d 11 c-C r e^{+}$mice had a much smaller spleen at d28p.i. in weight (Figure 4C) and cellularity
(Figure 4D), compared with their $\mathrm{Cre}^{-}$counterparts, suggesting that IRF-5 expression in $\mathrm{CD} 11 \mathrm{c}^{+}$cells is required to promote inflammation in the spleen following $L$. donovani infection. Moreover, myeloid cell numbers in L. donovani-infected CD11c$\mathrm{Cre}^{+}$mice were significantly reduced at d28 p.i. (Figure 4E). This lack of recruitment affected all myeloid cell populations (Supplemental Figures 3A-E). Similarly to Irf5 flox/flox $-C M V$ $\mathrm{Cre}^{+}$mice, reduced recruitment of inflammatory cells affected all cell populations equally; percentages of $\mathrm{B}, \mathrm{T}$, and myeloid cells did not vary between $\mathrm{CD} 11 \mathrm{c}-\mathrm{Cre}^{+}$and $\mathrm{Cr}^{-}$mice (data not shown).

Because the recruitment of monocytes has been associated with disease exacerbation (15), we were curious to know whether a dramatic reduction in inflammatory monocyte infiltration to the spleen would affect the parasite burden. As shown in Figure 4F, no significant differences in LDU were observed in the livers of infected $\mathrm{Cre}^{+}$and $\mathrm{Cr}^{-}$mice. In contrast, the splenic parasite burden was significantly lower in the absence of Irf5 expression in $\mathrm{CD}_{11 \mathrm{c}^{+}}$cells (Figure 4G).

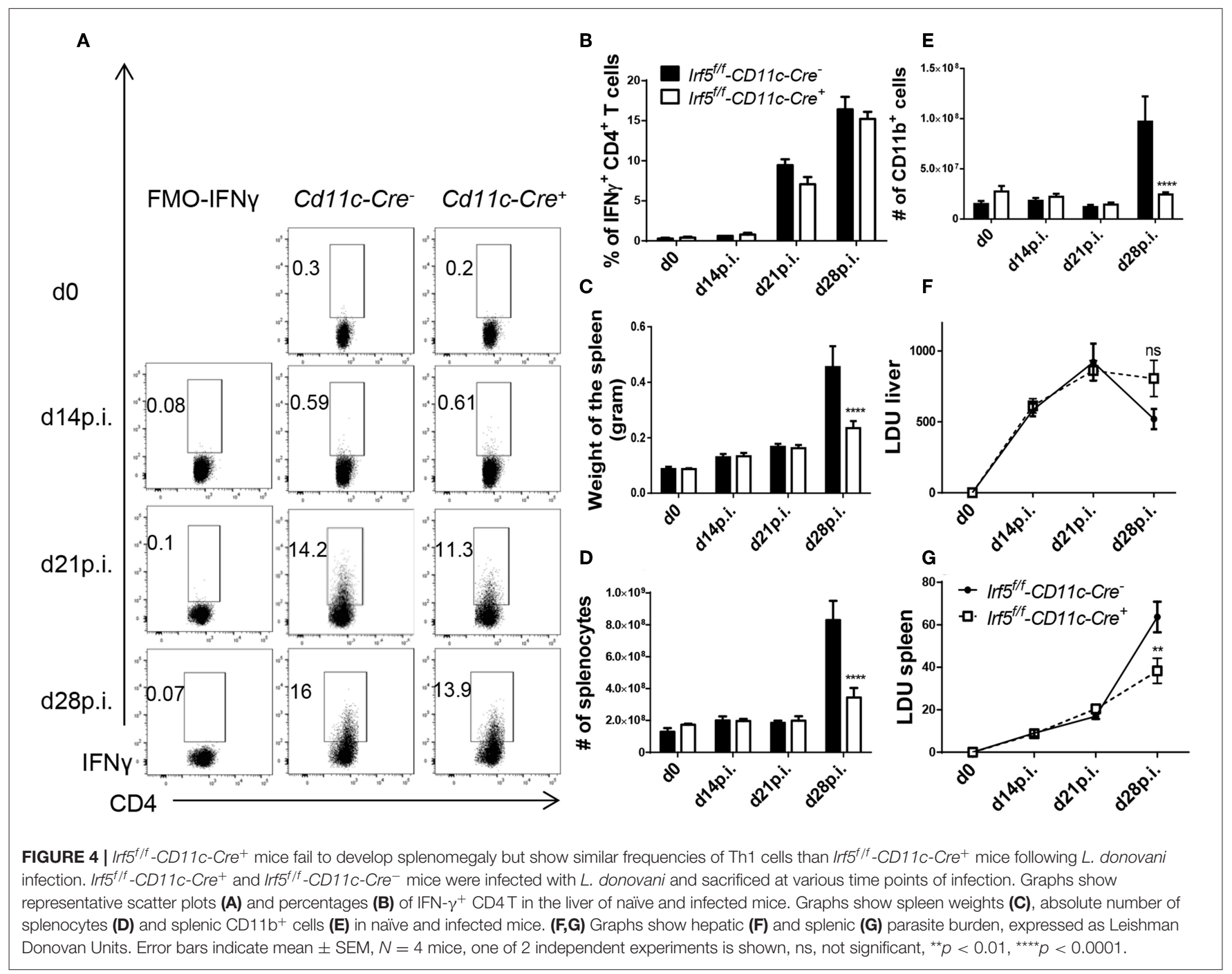


In summary, our results show that IRF-5-deficiency in $\mathrm{CD} 11 \mathrm{c}^{+}$cells severely affects inflammatory cell infiltration to the spleen, but does not hinder the development of Th1 responses in L. donovani-infected mice.

\section{DISCUSSION}

Splenomegaly and chronic inflammation are hallmark of visceral leishmaniasis and are associated with parasite persistence. IRF-5 is largely responsible for promoting inflammatory cell infiltration into the spleen, which ultimately results in splenomegaly. In the present study, we show that Irf5 expression in CD11c $\mathrm{c}^{+}$cells is involved in the induction of inflammation but is not necessary for development and/or maintenance of protective Th1 responses.

The LysM-Cre mouse model has successfully been used to study the role of specific genes in myeloid cells in the bone marrow (28), the lungs (29), the liver (30), the gut and the skin $(31,32)$. However, LysM-cre does not seem to be effective in deleting genes in splenic myeloid cells during VL. Our results are in agreement with Abram et al. that report deletion efficiency for LysM-cre of less than $40 \%$ in splenic macrophages and blood monocytes (33). Furthermore, analysis of mice with an insertion of EGFP into the $L y s M$ gene revealed that LysM is mostly highly expressed in granulocytes, especially neutrophils, followed by macrophages and only occasionally by monocytes (34). Thus, it is not surprising that this mouse model cannot be used to study gene functions in splenic myeloid cells following $L$. donovani infection, with exception of neutrophils. One should also take into account that deletion efficiency in blood monocytes is also very low and that this may change the interpretation of studies in disease models with extensive monocyte infiltrations, like cutaneous leishmaniasis for example. In an earlier publication, we have found that the CD11c-Cre mouse model is a better choice for deleting genes in splenic myeloid cells in the context of VL (14), because of the massive infiltration of inflammatory monocyte, which express high levels of CD11c during the chronic stage of disease. The current results confirm our previous data. Indeed, we observed a clear phenotype in CD11c-specific Irf5 $5^{-/}$mice after $L$. donovani infection, suggesting that Irf5 expression in neutrophils does not play a major role in the immunopathogenesis of VL.

Interestingly, $L$. donovani-infected CD11c-specific Irf5 $5^{-/-}$ mice display a massive impairment of myeloid cell recruitment to the spleen compared to IRF-5-sufficient mice. Splenomegaly results from the induction of emergency hematopoiesis, mainly during the chronic stages of $L$. donovani infection $(14,15)$. The fact that neutrophils, which are IRF-5 sufficient in our model, were also not recruited to the spleen suggest that IRF-5 may be required to drive inflammation/emergency hematopoiesis and/or for cell migration. In an experimental model of SLE, IRF-5 was reported to regulate the expression of CXCR4 and CCR2, two important molecules involved in monocyte migration (35). Moreover, IRF-5 is essential for maintaining proinflammatory $\mathrm{CD}_{11 \mathrm{c}^{+}}$macrophages/monocytes within lesions in an experimental atherosclerosis model (36). It is also possible that Irf5 expression in B cells may be involved in initiating inflammation. In fact, IRF-5 regulates activation, proliferation, differentiation and antibody production in human naïve $\mathrm{B}$ cells (37); moreover, Irf5 $5^{-/}$mice showed a reduction in hypergammaglobulinemia (38), which is required for sustaining inflammation during VL (39). Further investigations are needed to determine the functional role of this transcription factor in driving inflammation during VL. Taken together, our data demonstrate that a reduction in the recruitment of myeloid cells to the spleen during chronic infection results in heightened host resistance against $L$. donovani. These results are in agreement with the literature that reports a detrimental role for myeloid cells, particularly inflammatory monocytes, during $\operatorname{VL}(14,16,40)$.

We were surprised to observe that Th1 responses were not altered in myeloid cell-specific Irf5 $5^{-/-}$mice. Although T cell recruitment to the spleen was also impaired in CD11cspecific Irf5 $5^{-/}$mice during VL, the frequency of IFN $\gamma^{+}$ CD4 T cells was comparable to $\mathrm{Cr}^{+}$mice, suggesting that Irf5 expression in $\mathrm{CD}_{11 \mathrm{c}^{+}}$cells is not required for sustaining Th1 responses. This disagreement with the literature (27) could be explained by the fact that $\mathrm{CD} 4 \mathrm{~T}$ cell responses are primed very late during infection and peak at d21-28 p.i., when IRF-5 is not strongly expressed by myeloid cells. It is thus important to integrate knowledge on myeloid cell functions with the kinetic of $\mathrm{CD} 4 \mathrm{~T}$ cell priming and development in order to identify crucial molecules involved in these processes for a particular infection. IRF-5 expression in $\mathrm{CD}_{11 \mathrm{c}^{+}}$cells is not essential for Th1 priming during VL.

Because Th1 responses are thought to be essential for controlling parasite growth in leishmaniasis, it still remains unclear why the splenic parasite burden was lower in Cd11c$\mathrm{Cre}^{+}$mice compared to $\mathrm{Cr}^{-}$controls. One possible explanation is that monocytes, which act as safe houses during chronic VL (14-16), are recruited in lower numbers to the spleen. A second possibility that is not mutually exclusive with the first one is that CD8 T cells undergo stronger expansion in these mice. We have previously reported that IRF-5-mediated inflammation was required to induce HIF- $1 \alpha$ expression in dendritic cells and that this had an negative impact on their function and on $\mathrm{CD} 8 \mathrm{~T}$ cell expansion during the first 10 days of infection (6). It is thus possible that CD8 T cells underwent stronger expansion in infected Cd11c$\mathrm{Cre}^{+}$mice and that this contributed to enhanced parasite growth control.

In conclusion, we demonstrated that Irf5 expression in $\mathrm{CD} 11 \mathrm{c}^{+}$cells is required for the development of splenomegaly but does not affect the development or the maintenance of protective Th1 responses during experimental VL. Moreover, the Lysm-Cre mouse model should not be used to delete genes in splenocytes or inflammatory monocytes infiltrating the spleen.

\section{DATA AVAILABILITY STATEMENT}

All datasets generated for this study are included in the article/Supplementary Material. 


\section{ETHICS STATEMENT}

The animal study was reviewed and approved by Comité Institutionnel de Protection des Animaux of the INRS-Institut Armand Frappier.

\section{AUTHOR CONTRIBUTIONS}

LM designed and performed experiments, analyzed data, wrote the manuscript. MS, SS-B, and AF performed experiments and analyzed data. SS directed the study, interpreted data, and wrote the manuscript.

\section{REFERENCES}

1. Ready PD. Epidemiology of visceral leishmaniasis. Clin Epidemiol. (2014) 6:147-54. doi: 10.2147/CLEP.S44267

2. Kaye P, Scott P. Leishmaniasis: complexity at the host-pathogen interface. Nat Rev Microbiol. (2011) 9:604-15. doi: 10.1038/nrmicro2608

3. Bankoti R, Stäger S. Differential regulation of the immune response in the spleen and liver of mice infected with Leishmania donovani. J Trop Med. (2012) 2012:639304. doi: 10.1155/2012/639304

4. Charpentier T, Hammami A, Stäger S. Hypoxia inducible factor 1alpha: a critical factor for the immune response to pathogens and Leishmania. Cell Immunol. (2016) 309: 42-9. doi: 10.1016/j.cellimm.2016.06.002

5. Kaye PM, Svensson M, Ato M, Maroof A, Polley R, Stager S, et al. The immunopathology of experimental visceral leishmaniasis. Immunol Rev. (2004) 201:239-53. doi: 10.1111/j.0105-2896.2004.00188.x

6. Hammami A, Charpentier T, Smans M, Stäger S. IRF-5-Mediated inflammation limits CD8 $+\mathrm{T}$ cell expansion by inducing HIF-1alpha and impairing dendritic cell functions during leishmania infection. PLoS Pathog. (2015) 11:e1004938. doi: 10.1371/journal.ppat.1004938

7. Joshi T, Rodriguez S, Perovic V, Cockburn IA, Stäger S. B7-H1 blockade increases survival of dysfunctional CD8(+) T cells and confers protection against Leishmania donovani infections. PLoS Pathog. (2009) 5:e1000431. doi: 10.1371/journal.ppat.1000431

8. Hammami A, Abidin BM, Heinonen KM, Stäger S. HIF-1alpha hampers dendritic cell function and Th1 generation during chronic visceral leishmaniasis. Sci Rep. (2018) 8:3500. doi: 10.1038/s41598-018-21891-z

9. Fabié A, Mai LT, Dagenais-Lussier X, Hammami A, van Grevenynghe J, Stäger S. IRF-5 promotes cell death in CD4 T cells during chronic infection. Cell Rep. (2018) 24:1163-75. doi: 10.1016/j.celrep.2018. 06.107

10. Murphy ML, Cotterell SE, Gorak PM, Engwerda CR, Kaye PM. Blockade of CTLA-4 enhances host resistance to the intracellular pathogen, Leishmania donovani. J Immunol. (1998) 161:4153-60.

11. Maroof A, Beattie L, Zubairi S, Svensson M, Stager S, Kaye PM. Posttranscriptional regulation of II10 gene expression allows natural killer cells to express immunoregulatory function. Immunity. (2008) 29:295305. doi: 10.1016/j.immuni.2008.06.012

12. Bankoti R, Gupta K, Levchenko A, Stäger S. Marginal zone B cells regulate antigen-specific $\mathrm{T}$ cell responses during infection. J Immunol. (2012) 188:3961-71. doi: 10.4049/jimmunol.1102880

13. Ranatunga D, Hedrich CM, Wang F, McVicar DW, Nowak N, Joshi T, et al. A human IL10 BAC transgene reveals tissue-specific control of IL10 expression and alters disease outcome. Proc Natl Acad Sci USA. (2009) 106:17123-8. doi: 10.1073/pnas.0904955106

14. Hammami A, Abidin BM, Charpentier T, Fabié A, Duguay AP, Heinonen KM, et al. HIF- $1 \alpha$ is a key regulator in potentiating suppressor activity and limiting the microbicidal capacity of MDSC-like cells during visceral leishmaniasis. PLoS Pathog. (2017) 13:e1006616. doi: 10.1371/journal.ppat.1006616
ACKNOWLEDGMENTS

The authors thank the Canadian Institutes of Health Research (PJT-159647 to SS) for financial support. LM and MS were partly supported by a studentship from the Fondation Armand-Frappier.

\section{SUPPLEMENTARY MATERIAL}

The Supplementary Material for this article can be found online at: https://www.frontiersin.org/articles/10.3389/fimmu. 2019.03071/full\#supplementary-material

15. Abidin BM, Hammami A, Stäger S, Heinonen KM. Infection-adapted emergency hematopoiesis promotes visceral leishmaniasis. PLoS Pathog. (2017) 13:e1006422. doi: 10.1371/journal.ppat.1006422

16. Terrazas C, Varikuti S, Oghumu S, Steinkamp HM, Ardic N, Kimble J, et al. Ly6C(hi) inflammatory monocytes promote susceptibility to Leishmania donovani infection. Sci Rep. (2017) 7:14693. doi: 10.1038/s41598-017-14935-3

17. Barnes BJ, Moore PA, Pitha PM. Virus-specific activation of a novel interferon regulatory factor, IRF-5, results in the induction of distinct interferon alpha genes. J Biol Chem. (2001) 276:23382-90. doi: 10.1074/jbc.M101216200

18. Takaoka A, Yanai H, Kondo S, Duncan G, Negishi H, Mizutani T, et al. Integral role of IRF-5 in the gene induction programme activated by Toll-like receptors. Nature. (2005) 434:243-9. doi: 10.1038/nature03308

19. Graham RR, Kozyrev SV, Baechler EC, Reddy MV, Plenge RM, Bauer JW, et al. A common haplotype of interferon regulatory factor 5 (IRF5) regulates splicing and expression and is associated with increased risk of systemic lupus erythematosus. Nat Genet. (2006) 38:550-5. doi: 10.1038/ng1782

20. Dieguez-Gonzalez R, Calaza M, Perez-Pampin E, de la Serna AR, FernandezGutierrez B, Castañeda S, et al. Association of interferon regulatory factor 5 haplotypes, similar to that found in systemic lupus erythematosus, in a large subgroup of patients with rheumatoid arthritis. Arthritis Rheum. (2008) 58:1264-74. doi: 10.1002/art.23426

21. Carmona FD, Martin JE, Beretta L, Simeón CP, Carreira PE, Callejas JL, et al. The systemic lupus erythematosus IRF5 risk haplotype is associated with systemic sclerosis. PLoS ONE. (2013) 8:e54419. doi: 10.1371/journal.pone.0054419

22. Kristjansdottir G, Sandling JK, Bonetti A, Roos IM, Milani L, Wang C, et al. Interferon regulatory factor 5 (IRF5) gene variants are associated with multiple sclerosis in three distinct populations. J Med Genet. (2008) 45:3629. doi: 10.1136/jmg.2007.055012

23. Paun A, Bankoti R, Joshi T, Pitha PM, Stäger S. Critical role of IRF-5 in the development of T helper 1 responses to Leishmania donovani infection. PLoS Pathog. (2011) 7:e1001246. doi: 10.1371/journal.ppat.1001246

24. Smelt SC, Engwerda CR, McCrossen M, Kaye PM. Destruction of follicular dendritic cells during chronic visceral leishmaniasis. J Immunol. (1997) 158:3813-21.

25. Silva-Barrios S, Smans M, Duerr CU, Qureshi ST, Fritz JH, Descoteaux A, et al. Innate immune B cell activation by leishmania donovani exacerbates disease and mediates hypergammaglobulinemia. Cell Rep. (2016) 15:242737. doi: 10.1016/j.celrep.2016.05.028

26. Purtha WE, Swiecki M, Colonna M, Diamond MS, Bhattacharya D. Spontaneous mutation of the Dock2 gene in Irf5-/- mice complicates interpretation of type I interferon production and antibody responses. Proc Natl Acad Sci USA. (2012) 109:E898-904. doi: 10.1073/pnas.1118155109

27. Krausgruber T, Blazek K, Smallie T, Alzabin S, Lockstone H, Sahgal N, et al. IRF5 promotes inflammatory macrophage polarization and TH1-TH17 responses. Nat Immunol. (2011) 12:231-8. doi: 10.1038/ni.1990

28. Imtiyaz HZ, Williams EP, Hickey MM, Patel SA, Durham AC, Yuan LJ, et al. Hypoxia-inducible factor 2alpha regulates macrophage function in mouse 
models of acute and tumor inflammation. J Clin Invest. (2010) 120:2699714. doi: 10.1172/JCI39506

29. McCubbrey AL, Allison KC, Lee-Sherick AB, Jakubzick CV, Janssen WJ. Promoter specificity and efficacy in conditional and inducible transgenic targeting of lung macrophages. Front Immunol. (2017) 8:1618. doi: 10.3389/fimmu.2017.01618

30. Alzaid F, Lagadec F, Albuquerque M, Ballaire R, Orliaguet L, Hainault I, et al. IRF5 governs liver macrophage activation that promotes hepatic fibrosis in mice and humans. JCI Insight. (2016) 1:e88689. doi: 10.1172/jci.insight. 88689

31. Andrianne M, Assabban A, La C, Mogilenko D, Salle DS, Fleury S, et al. Tristetraprolin expression by keratinocytes controls local and systemic inflammation. JCI Insight. (2017) 2:92979. doi: 10.1172/jci.insight.92979

32. Malik A, Sharma D, Malireddi RKS, Guy CS, Chang TC, Olsen $\mathrm{SR}$, et al. SYK-CARD9 signaling axis promotes gut fungi-mediated inflammasome activation to restrict colitis and colon cancer. Immunity. (2018) 49:515-30 e5. doi: 10.1016/j.immuni.2018. 08.024

33. Abram CL, Roberge GL, Hu Y, Lowell CA. Comparative analysis of the efficiency and specificity of myeloid-Cre deleting strains using ROSA-EYFP reporter mice. J Immunol Methods. (2014) 408:89-100. doi: 10.1016/j.jim.2014.05.009

34. Faust N, Varas F, Kelly LM, Heck S, Graf T. Insertion of enhanced green fluorescent protein into the lysozyme gene creates mice with green fluorescent granulocytes and macrophages. Blood. (2000) 96:71926. doi: 10.1182/blood.V96.2.719.014k29_719_726

35. Yang L, Feng D, Bi X, Stone RC, Barnes BJ. Monocytes from Irf5-/mice have an intrinsic defect in their response to pristane-induced lupus. J Immunol. (2012) 189:3741-50. doi: 10.4049/jimmunol.12 01162
36. Seneviratne AN, Edsfeldt A, Cole JE, Kassiteridi C, Swart M, Park I, et al. Interferon regulatory factor 5 controls necrotic core formation in atherosclerotic lesions by impairing efferocytosis. Circulation. (2017) 136:1140-54. doi: 10.1161/CIRCULATIONAHA.117.027844

37. De S, Zhang B, Shih T, Singh S, Winkler A, Donnelly R, et al. B cellintrinsic role for IRF5 in TLR9/BCR-induced human B cell activation, proliferation, and plasmablast differentiation. Front Immunol. (2017) 8:1938. doi: 10.3389/fimmu.2017.01938

38. Feng D, Yang L, Bi X, Stone RC, Patel P, Barnes BJ. Irf5-deficient mice are protected from pristane-induced lupus via increased Th2 cytokines and altered IgG class switching. Eur J Immunol. (2012) 42:147787. doi: $10.1002 /$ eji.201141642

39. Silva-Barrios S, Stäger S. Hypergammaglobulinemia sustains the development of regulatory responses during chronic Leishmania donovani infection in mice. Eur J Immunol. (2019) 49:1082-91. doi: 10.1002/eji.201847917

40. Abidin BM, Owusu Kwarteng E, Heinonen KM. Frizzled-6 regulates hematopoietic stem/progenitor cell survival and self-renewal. J Immunol. (2015) 195:2168-76. doi: 10.4049/jimmunol.1403213

Conflict of Interest: The authors declare that the research was conducted in the absence of any commercial or financial relationships that could be construed as a potential conflict of interest.

Copyright (0) 2020 Mai, Smans, Silva-Barrios, Fabié and Stäger. This is an open-access article distributed under the terms of the Creative Commons Attribution License (CC $B Y)$. The use, distribution or reproduction in other forums is permitted, provided the original author(s) and the copyright owner(s) are credited and that the original publication in this journal is cited, in accordance with accepted academic practice. No use, distribution or reproduction is permitted which does not comply with these terms. 